\title{
OBSERVATION, MODELLING AND CURE OF TRANSVERSE INSTABILITIES AT THE ESRF
}

\author{
J. L. Revol, R. Nagaoka, ESRF, Grenoble, France
}

\begin{abstract}
Multibunch operation at the ESRF is affected by transverse resistive-wall instabilities in horizontal and vertical planes. Chromaticities are shifted to positive values and a partial filling is used to raise the instability thresholds. Systematic measurements have been carried out to characterise the instabilities as a function of the filling pattern, chromaticity and RF voltage. A beambased modelling of the impedance has been made, using combined BBR and resistive wall impedance. For single bunch, the mechanism that drives the vertical instabilities is presented, while for horizontal ones recent observations are discussed. Incoherent betatron tune shifts with the average beam current, observed in both plane but with opposite polarities, have been investigated for single and multibunch. First results from preliminary tests on a new hybrid mode are discussed. Besides resistive-wall instabilities, ion trapping as well as fast ion-beam blow up have been observed. The effectiveness of a mode-bymode transverse feedback to damp the instabilities is also presented.
\end{abstract}

\section{INTRODUCTION}

The ESRF is routinely operated in multibunch at 200 $\mathrm{mA}$, single bunch up to $20 \mathrm{~mA}$ and in few bunch modes, like $90 \mathrm{~mA}$ in 16 bunches. A continuous effort has been put on the experimental and theoretical study of single and multibunch beam instabilities (both longitudinal and transverse), with the improvement of operation as a primary goal. Over the last years, the machine impedance had continuously evolved (installation of a third pair of cavities, installation of low gap vacuum chambers, partial conversion from stainless steel to aluminium or copper coating,...). Moreover, the changes in the optics (low vertical beta function, reduction of the emittances,..) and the continuous improvement of diagnostics emphasise the analysis of transverse instabilities. Recently, the study of a new hybrid mode has confirmed the necessity of a global approach for single/multi bunch instability studies.

\section{MULTIBUNCH ISSUES}

Horizontal and vertical resistive wall (RW) impedance affects multibunch operation. With an increasing number of low gap vacuum chambers for insertion devices, the effects continuously increase from the start-up of the machine. Nevertheless, the on-going replacement of stainless steel by aluminium or copper for the realisation of newly installed vacuum has had a positive impact on this evolution.

\subsection{Instability threshold}

Only the first five low frequency couple bunch modes are concerned by the long range wake field generated by the RW impedance. Shifting the chromaticity $(\xi)$ to positive value and adopting a partial filling raises the current threshold. With the increased chromaticity (Fig 1), the stabilising effect of the broadband impedance is enhanced (the beam spectrum interacts with a larger positive real part of the impedance). For vertical, the simulation of the current threshold including both vertical BBR (estimated from mode merging, see 2.1) and RW impedance are in good agreement with the experience. Due to the unavailability of a good BBR model for horizontal, this has not been performed for horizontal. Moreover, with increased $\xi$ and high current the interaction of higher, head-tail modes (vertical mode -2 at $200 \mathrm{~mA}$ in uniform) added additional stability. (Fig.2 right).

The strong stabilising effect of the filling pattern is also illustrated in fig 1 . At $100 \mathrm{~mA}$, the horizontal chromaticity could be reduced to zero in a non flat $1 / 3$ filling while it has be pushed to 0.14 for uniform. This additional stabilisation, which is also observed in vertical, is anticipated to come from the spread in betatron frequency induced by the incoherent betatron tune shift provided by the asymmetry of the vacuum chamber [see2.4] [1].
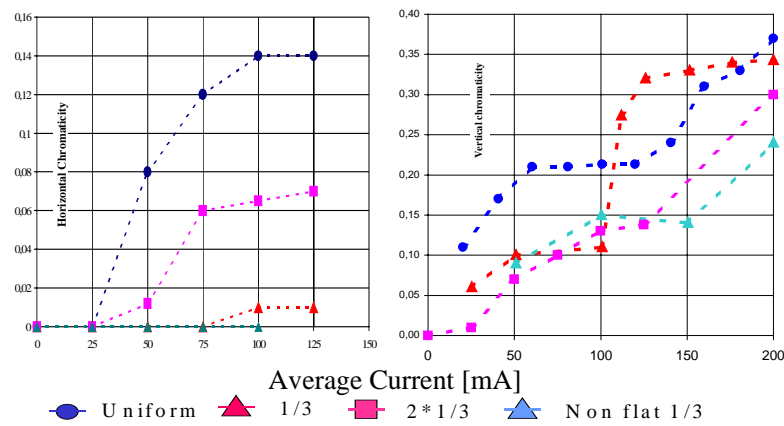

ent $[\mathrm{mA}]$

Figure 1: Horizontal $\xi$ (left) and vertical (right) $\xi$. at the current threshold for different filling pattern.

Horizontal and vertical beam ion instabilities (BII and fast BII) have been observed in uniform and partial filling patterns (Fig.4) [2]. The presence of BII slightly complicates the analysis of vertical RW instabilities for which the beam emittance is an excellent diagnostics. For operation, BII are also stabilised by the increased $\xi$.

\subsection{Transverse Feedback}

A mode by mode feedback acting on the five first coupled bunch modes driven by the vertical resistive wall impedance has been installed. It has been successfully 
tested in uniform and in $2 * 1 / 3$ filling, allowing $200 \mathrm{~mA}$ with a zero $\xi$. Nevertheless, the BII observed in uniform make it incompatible with the low emittance at very low chromaticity. At $200 \mathrm{~mA}$ in $2 * 1 / 3$, fast BII exhibit excited mode around 7 revolution frequency (F0) at zero $\xi$ (fig.2 up). But the increase to a slight positive value totally damps it (Fig2.low), resulting in a perfectly flat spectrum with only three corrected modes, restoring both the emittance and the lifetime. The feedback will be extended to two horizontal modes in order to reduce the horizontal chromaticity also close to zero. Nevertheless, the lifetime which is in the range of 60 hours makes the feedback useless for operation for the time being. It will mainly be used for machine physic studies at low chromaticity and for growth rate measurement with the gating function.

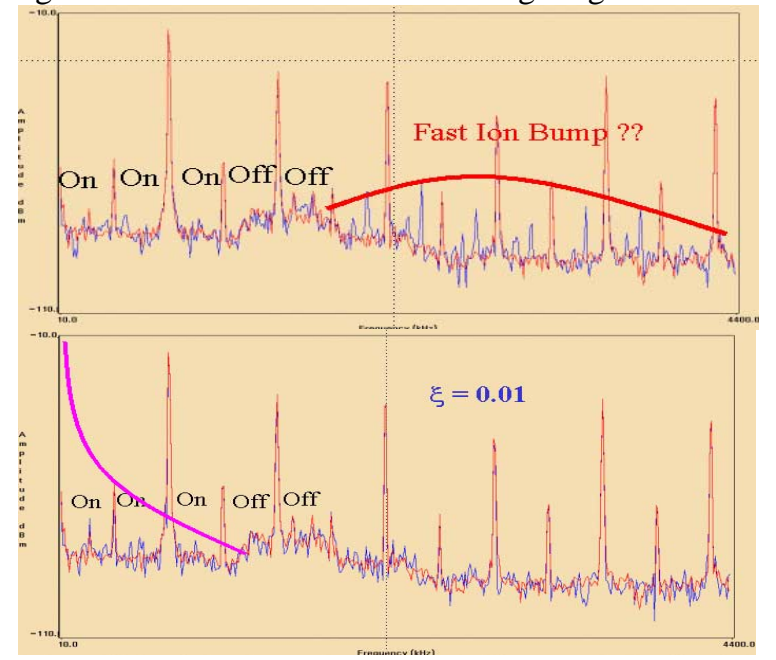

Figure 2: Vertical CB spectrum up to $12 \mathrm{~F} 0$ in the $2 * 1 / 3$ filling at zero vertical $\xi$ (up) and a slight positive (bottom) (red lines corresponds to the multiple of F0)

\subsection{Multibunch detuning}

Betatron tune shift with the average current (Fig 3) has been extensively studied this year. This quadrupolar effect is supposed to be induced by a long range field effect imposed by the non-axis symmetry of the RW vacuum chamber [for more information, see reference1].

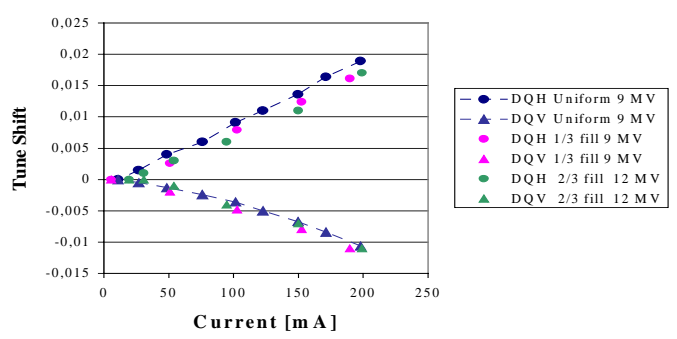

Figure 3: Betatron tune shift with current.

\section{SINGLE BUNCH ISSUES}

Single bunch operation at the ESRF is severely limited by transverse instabilities. Vertical instabilities, which are more critical, were extensively studied within the frame of a $\mathrm{PhD}$ work [3], while the horizontal regime has just been initiated.

\subsection{Zero chromaticity regime}

The transverse mode coupling instability (TMCI) between mode 0 and mode -1 sets a severe current limitation at nearly zero $\xi$ (Figs. 4). The horizontal instability (Ith $=2 \mathrm{~mA}$ ) can no longer be regarded as weak as compared to vertical ( $0.8 \mathrm{~mA})$, and investigation of the driving impedance appeared worthwhile.
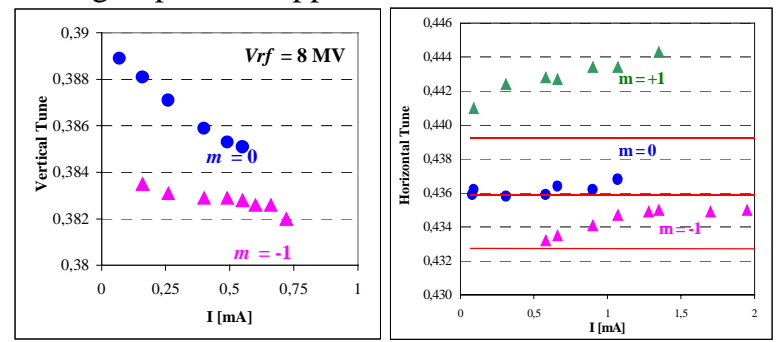

Figure 4: Detuning of head-tail mode 0 and -1 measured in the mode-merging regime, for vertical (left) and for horizontal (right) at $\mathrm{Vrf}=8 \mathrm{MV}$ (at slightly positive chromaticity in order to see mode -1 and mode +1 ).

Vertical observations, which show a strong detuning of mode 0 , are in accordance with the TMCI theory. A fit of the mode detuning as well as the threshold in frequencycurrent plane fixes the parameters of the BBR (BroadBand Resonator) impedance. A shunt impedance of $R_{T}=$ 6.5 $\mathrm{M} \Omega / \mathrm{m}$ and a resonant frequency of $f_{r}=22 \mathrm{GHz}$ are derived assuming a quality factor $Q$ of 1 [3]. It should be stressed here that the fact that mode -1 is defocused, on top of mode 0 , is mainly responsible for $f_{r}$ becoming as high as $22 \mathrm{GHz}$. For horizontal, a few attempts to fit a BBR impedance turned out unsuccessful in reproducing the observed combination of no detuning of mode 0 and a strong focusing of modes \pm 1 .

\subsection{High current regime}

In both planes, the chromaticity shift to positive values avoids hitting the TMCI and increases the current threshold non-linearly (Fig. 5 and 6). Thanks to the bunch lengthening with current and the chromaticity, more stable head-tail modes interact with the real negative impedance in the negative frequency.

For vertical, it has been shown that the incoherent frequency spread induced by longitudinal instabilities is large enough to entirely stabilise and overcome the headtail regime. In order to reproduce the observed threshold, a post head-tail mechanism was introduced for chromaticities above 0.2 . The post head-tail regime is stabilised by the spread in energy, which induces an incoherent dispersion of the betatron frequency [3]. In that case, the growth time of the instability becomes shorter than the synchrotron period and the notion of modes is invalidated. This is shown by the monotonous curve which gives the tune at the threshold

For horizontal (Fig. 6), the instability mechanism is still evolving in the head-tail regime. This is mainly illustrated by the step function on the threshold curve (head-tail modes). The focusing effect initiated in the mode merging regime is confirmed for the head tail regime 

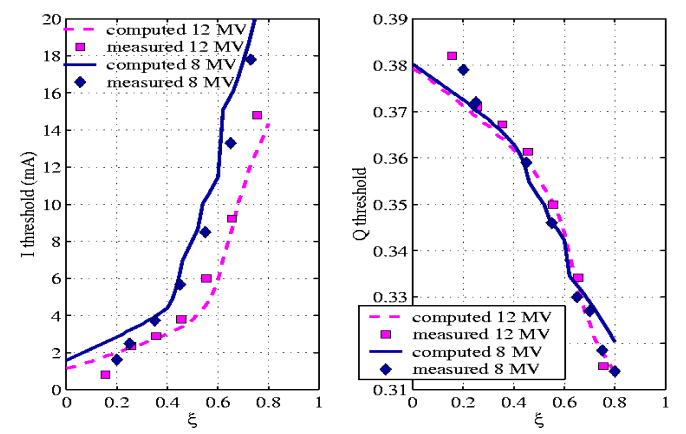

Figure 5: Current threshold (left) and. tune at the threshold (right) as a function of the vertical $\xi$.
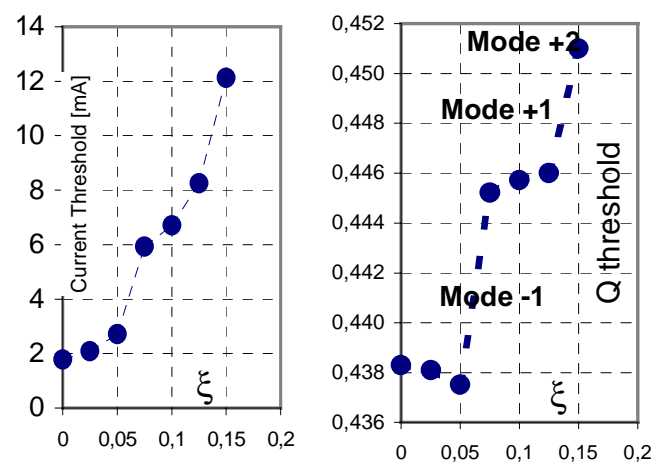

Figure 6: Current threshold (left) and tune at the threshold (right) as a function of the horizontal $\xi$.

\subsubsection{Horizontal focusing effect:}

The resistive-wall wake field that induces the multibunch detuning (betatron tune shifts with current), also gives a significant incoherent tune shift in single bunch, due to its short range part [1]. This incoherent effect, which is negligible in the vertical plane, should be in the range of $\Delta \mathrm{Qx}=0.002 / \mathrm{mA}$ for horizontal, due to the large horizontal beta function. The subtraction of this incoherent detuning results in a more consistent record for the mode merging. At larger chromaticities, it should transform the observed focusing effect in a defocusing effect and consequently representing negative modes instead of positive ones. Further analysis is under investigation in order to extract the incoherent detuning and to estimate the horizontal impedance.

\section{HYBRID FILLING ISSUES}

A new hybrid mode of operation ( 24 groups of 8 bunches with a single bunch in the middle), which could serve different user communities, is under investigation (Fig. 7). During preliminary tests (Fig.8), the single bunch was first filled at $7.3 \mathrm{~mA}$ and then the current was topped-up to $200 \mathrm{~mA}$ in a group of bunches. At a given current, it was observed that the stored single bunch started to decrease. A slight decrease of the horizontal tune stopped these phenomena. The single bunch alone experiments an increase of the incoherent tune as a function of the beam current. The detuning is much larger for the $7 \mathrm{~mA}$ bunch than for the others which are only filled at $1 \mathrm{~mA}$. In the case of the hybrid mode, the single bunch experiments both multibunch and single bunch detuning effects, and consequently, the vicinity of the resonance is then more dramatic for the single bunch. The working point in the tune diagram is a key point for this new filling. Moreover, the cleaning based on the betatron excitation of the low populated bunches enhanced this difficulty.

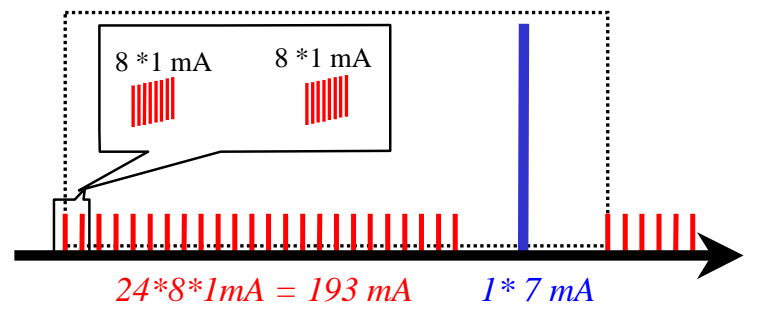

Figure 7: New hybrid mode for the ESRF.

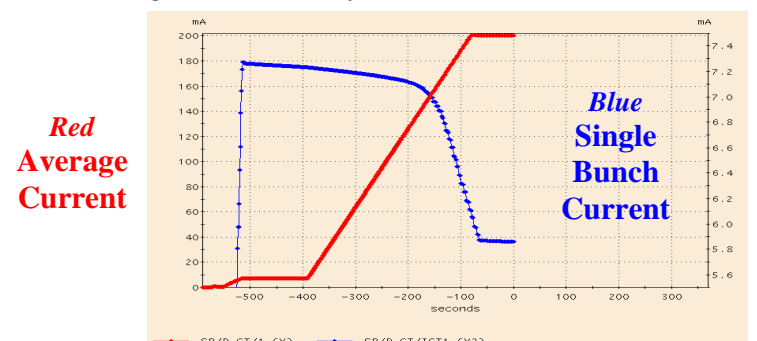

Figure 8: Single Bunch Current Loss (Due to H focusing).

\section{CONCLUSION}

In analogy to the study of vertical single bunch instabilities, in which an important link to the longitudinal dynamics was established, transverse dynamics, whether single or multibunch, are under a strong influence of both RW and BBR impedance, which are usually decoupled. In particular, we have seen that the RW of the low-gap chambers create a mean field, in addition to the dipolar wake, which perturbs the beam in both the short and long range. Without an overall knowledge of the effects concerned, the correct interpretation of the observations seems difficult. Further investigation, both theoretical and experimental, is in progress to clarify the different impacts of the impedance. As well, BII, which could affect the emittance, will be explored in more detail.

\section{ACKNOWLEDGEMENT}

The authors would like to thank E. Plouviez for his contribution to this study. They are also grateful to $\mathrm{J}$ Jacob, G Besnier and P Kernel for their participation and to N. Michel for feedback development.

\section{REFERENCES}

[1] R Nagaoka, "Impact of Resistive Wall wake fields generated by low gap chambers on the beam at the ESRF.", This conference.

[2] R. Nagaoka, J L Revol, J Jacob, “Observation, Analysis and Cure of Transverse Multibunch Instabilities at the ESRF", EPAC2000.

[3] P. Kernel, J.L. Revol, R. Nagaoka, G. Besnier, "High Current Single Bunch Transverse Instabilities at the ESRF: A new Approach?", EPAC 2000, Vienna. 